\title{
Relativistic Astrophysics in Uzbekistan
}

\author{
Bobomurat Ahmedov ${ }^{1,2}$ \\ ${ }^{1}$ Ulugh Beg Astronomical Institute, Astronomicheskaya 33, Tashkent 100052, Uzbekistan \\ ${ }^{2}$ National University of Uzbekistan, Tashkent 100174, Uzbekistan \\ email: ahmedov@astrin.uz
}

\begin{abstract}
During the last twenty years, due to the extensive help and assistance of the international scientific community, there has been a great success in the development and establishment of new well-functioning and competitive scientific groups specialized in general relativity and relativistic astrophysics in Uzbekistan (Tashkent), Kazakhstan (Astana and Almaty), Kyrgyzstan (Bishkek) and great achievements have been made on the study in Central Asia in relativistic cosmology and astrophysics of compact gravitational objects.
\end{abstract}

Keywords. Relativistic astrophysics, Central Asia, black holes, neutron stars.

\section{Introduction}

The main fields in which the scientific activity on relativistic astrophysics in Uzbekistan has concentrated are those related to the development of applications of general relativity and gravitation (GR) to relativistic astrophysics. These include relativistic astrophysics and cosmology, gravitational waves and practical results on ionospheric studies for satellite based positioning systems.

Relativistic Astrophysics. The past decades have seen spectacular new revolutionary discoveries and developments in our knowledge of cosmology, the physics of compact objects and in high precision gravity experiments. As a result, relativistic astrophysics and cosmology have become a very attractive and modern area of research in Central Asia.

Neutron Stars and Pulsars. Compact relativistic neutron stars with the strong gravitational and electromagnetic fields observed as pulsars play a role as an astrophysical laboratory, which provides the best strong-field test of general relativity, and it is the subject of current research in Central Asia.

Black Holes. Starting from being a purely mathematical abstraction, over the past few decades black holes (BHs) have become one of the most interesting and attractive objects for analytical, numerical and observational research. BHs are essentially generalrelativistic objects and require the use of the complex mathematical apparatus of general relativity for a complete description of the physical phenomena taking place in their vicinity, thus making the physics of BHs particularly challenging in Central Asia. Recently the study of BHs and, in particular, BH binary systems, has attracted considerable new interest as these are now considered as the most probable candidates for gravitational wave detection. When two astrophysical BHs merge, they release an enormous amount of energy in the form of gravitational radiation, making them the brightest objects in the Universe. In view of the rapid scientific progress in this direction, it is now of crucial importance for a search to be made for possible electromagnetic phenomena, following each phase of $\mathrm{BH}$ binary evolution. Besides the astrophysical importance for the complete physical description of $\mathrm{BH}$ binary evolution, the presence of a detectable electromagnetic 
signal from the BH binary merger would provide an additional channel of information, which would help us to interpret correctly measurements of the gravitational signal.

The relativistic astrophysics group in Tashkent has long-standing experience in applying general relativity to astrophysical problems and in the use of state of the art numerical and analytical techniques in studying the electrodynamics of relativistic stars and black holes. In particular, the basic formalism to study the influence of strongly curved spacetime on the properties of interior and exterior electromagnetic fields of magnetized relativistic stars and black holes has been developed. The force-free magnetosphere of oscillating and rotating magnetized neutron stars has been also studied. A qualitative model for the explanation of the phenomenology of intermittent pulsars in terms of stellar oscillations that are periodically excited by star glitches has been proposed. The conditions for radio emission in rotating and oscillating magnetars, by focusing on the main physical processes determining when the magnetars may be radio-loud or radio-quiet are also studied. Present observations showing a close connection between the burst activity of magnetars and the generation of the radio emission in the magnetar magnetosphere are naturally accounted for. A general formalism to describe the black hole shadow as an arbitrary polar curve expressed in terms of a Legendre expansion has been developed, and it has been shown that the new formalism provides an accurate and robust description of noisy observational data, with smaller error variances when compared to previous measurements of the distortion. Gravitational lensing by the various compact objects has been extensively studied. Here I will describe some parts of the current theoretical research in relativistic astrophysics under progress in Uzbekistan.

\section{Rotating black hole solutions}

In Toshmatov et al. (2014) a regular black hole solution with the source involving nonlinear electrodynamics as obtained by Ayón-Beato and García (ABG, Ayón-Beato \& A. García 1998) is used to generate a rotating regular black hole solution, which includes the ABG and Kerr metrics as special cases. The relation between the Einstein vacuum solution and any nonvacuum solution of general relativity opens new directions in studying the properties of the new solution with a nonlinear electrodynamic source. Obviously, when the electric charge vanishes, the solution reduces to the vacuum one. Moreover, it has been shown that the inclusion of the rotation into spacetime violates the weak energy condition inside the rotating regular ABG black hole. The solution obtained gives an opportunity to study the geometrical and causal structures, as well as to test particle motion around the rotating regular black hole, which had later been studied in Toshmatov et al. (2015a).

In Toshmatov et al. (2017a) a rotating quintessential black hole solution is constructed by using the modified Newman-Janis Algorithm (NJA). For simplicity, we studied in detail properties of the rotating quintessential black hole solution with the special value of the quintessential parameter $\omega_{q}=-2 / 3$. Calculations have shown that in the rotating case the upper limit on the quintessence parameter $c$ corresponding to the black hole spacetimes is $c \leqslant 1 / 6$, which is significantly larger in comparison with the nonrotating case when the limit reads $c \leqslant 1 / 8$ - see Younas et al. (2015). Calculations have shown that the static radius limiting existence of circular geodesics does not depend on the rotation parameter of the spacetime. The limits on the dimensionless parameters $a$ and $c$ of the quintessential rotating spacetimes are given. The regions of the stable circular geodesics are determined for both the black hole and naked singularity spacetimes.

In Toshmatov et al. (2017b) the generic rotating black hole solutions in general relativity coupled to nonlinear electrodynamics were introduced. We thus generalized the spherically symmetric black hole solution obtained by Fan \& Wang (2016) using the method based on the alternate NJA. In our model one can construct exact black hole 
solutions by choosing appropriate values for the characteristic parameters $\mu$ and $\nu$ as in the case of the nonrotating case, when the known solutions can be obtained for fixed values of the parameters: $\nu=2-$ Bardeen-like black holes, $\nu=3$ - Hayward-like black holes, and $\nu=1-$ a new type of black hole solution which approaches the Maxwell field in a weak field limit. Moreover, we have shown that some main properties of these rotating black holes are almost the same as those occurring in the nonrotating case which was discussed in Fan \& Wang (2016). Especially, in the case of zero gravitational mass black hole, $M=0$, the $\mu \geqslant 3$ spacetimes are the regular (nonsingular) ones. Furthermore, we have shown that the presence of the gravitational mass does not affect the energy conditions and the fact that the obtained solutions violate the weak and strong energy conditions close to the center of the spacetime. Though an increase in the value of $\mu$ decreases the depth of the violation of energy conditions, the violations are always preserved even for the large values of $\mu$.

\section{Quasinormal modes of spherically symmetric black holes}

In Toshmatov et al. (2016) the scalar, electromagnetic, axial and polar gravitational perturbations of the Reissner-Nordström-like black hole with tidal charge in the Randall-Sundrum braneworld have been studied. By using the sixth order WentzelKramers-Brillouin (WKB) method, we have calculated the quasinormal frequencies of these perturbations. Results have shown that with increasing tidal charge parameter, the frequency of the real oscillations decreases while damping rate increases, unlike the case of the standard Reissner-Nordström black hole. Moreover, it has been shown that the current black hole solution localized in the Randall-Sundrum braneworld is stable against scalar, electromagnetic and gravitational perturbations.

In Toshmatov et al. (2015b) quasinormal modes of the linear "axial" scalar, electromagnetic and gravitational perturbations in the Hayward, Bardeen and ABG regular black hole spacetimes by using the sixth order WKB approximation have been studied. Calculations have shown that increasing the spacetime charge parameter implies monotonic increasing of the real part of quasinormal frequency and monotonic decreasing of the imaginary part of quasinormal frequency, i.e., the damping rate of the wave decreases. It means that in the regular black holes oscillators are better (slowly damped) than in the field of Schwarzschild black holes. In the Hayward and Bardeen regular black hole spacetimes, damping of the quasinormal modes is always largest for the scalar fields, mediate for the electromagnetic fields and smallest for the gravitational fields. However, for the ABG black hole spacetimes the situation is more complex, as the scalar fields have damping smaller than the electromagnetic fields, if the charge parameter of these spacetimes $d>0.56$. It has been shown that all of the considered regular black holes are stable against linear "axial" gravitational, scalar and electromagnetic perturbations.

In Toshmatov et al. (2017c) the dynamics of massive or massless scalar fields in the Schwarzschild-de Sitter spacetimes has been studied. First, we have given the lower limit on the value of the mass of the scalar field for the waves with quasinormal frequencies related to the effective potential barrier between the black hole and the cosmological horizons of the Schwarzschild-de Sitter spacetime, and thus enabling the wave to reach an observer at infinity without meeting any other potential wall. Surprisingly, the critical mass of the scalar field determining the lower limit depends only on the dimensionless cosmological parameter given by the relation $y=\Lambda M^{2} / 3$ where $M$ is the mass of the black hole, $\Lambda$ is the cosmological constant. Moreover, the same statement is relevant to the Reissner-Nordström-de Sitter spacetimes.

Secondly, we have determined the quasinormal frequencies of the oscillations in the eikonal approximation related to the unstable photon circular orbits and shown that with increasing cosmological parameter $y$ of the Schwarzschild-de Sitter spacetime, both the frequency of the oscillations of the quasinormal modes and the damping rate decrease. 
In Toshmatov et al. (2017d) scalar and electromagnetic perturbations in the family of singularity-free nonrotating black-hole spacetimes found in Bambi, Modesto \& Rachwal (2017); Modesto \& Rachwal (2016) have been studied. We found that the scalar perturbations depend on the scaling factor $S$, while the electromagnetic perturbations in a spherically symmetric black-hole spacetime are not affected by a conformal transformation of the background metric. We had thus focused our study on scalar perturbations, since the electromagnetic perturbations are the same as in the Schwarzschild metric and they have been already studied by other authors. We found that the singularity-free nonrotating black holes in conformal gravity are stable under scalar and electromagnetic perturbations (the case of electromagnetic perturbations directly follows from the fact that it is true in the Schwarzschild spacetime). The quasinormal mode spectrum of scalar perturbations depends on the parameters $L$ and $N$. If we assume that the quasinormal mode spectrum for scalar perturbations is not too different from that of gravitational waves we can argue that the detection of quasinormal modes from astrophysical black holes can constrain the parameters $L$ and $N$. Note that for $\ell=2$ (which should be the dominant mode in the case of the coalescence of a binary black hole merging into a single black hole) the impact of $L$ and $N$ can be large and surely detectable. Our calculations cannot be directly applied to the recent observations of gravitational waves by the LIGO experiment because here we have only studied the case of nonrotating black holes.

In Toshmatov et al. (2018) the formalism to construct the electrically and magnetically charged (singular and regular) black-hole solutions in general relativity coupled to the nonlinear electrodynamics has been developed. For our special interest, we constructed the family of new singular nonlinear electrodynamics black-hole solutions which tends to the linear (Maxwell) electrodynamics in the weak field limit, based on the Lagrangian density supposed in Fan \& Wang (2016). We showed that these solutions are singular at $r=0$ and unlike the other standard singular solutions, these solutions are convertible to the regular ones by the special condition: $M=q^{3} / \alpha$ where $\alpha$ is constant parameter in the unit of length squared. As is usual with regular black-hole spacetimes, these Maxwellian regular black-hole spacetimes also represent black hole, extremal black hole or no-horizon spacetimes, depending on the values of the gravitational mass and nonlinear electrodynamic field parameters.

The study is dedicated to the axial electromagnetic perturbations of the general nonlinear electrodynamic black-hole solutions considering the electromagnetic perturbations that do not alter the spacetime geometry. We showed that the electromagnetic perturbations of the nonlinear electrodynamic black holes give different potentials and, consequently, different results for the quasinormal frequencies, as compared to those related to the Reissner-Nordström black holes in the standard electrovacuum theory. It is well known that the electromagnetic perturbations of the electrically and magnetically charged black holes in linear electrodynamics (Reissner-Nordström) are isospectral, i.e., they have the same effective potentials and quasinormal frequencies, however, in the case of the nonlinear electrodynamic black holes, electrically and magnetically charged black holes have different potentials and different quasinormal modes spectra. As a special case, we calculated quasinormal modes of the magnetically charged Maxwellian regular black hole with $\mu=3$ and compared them with the ones of the Reissner-Nordström black holes by normalizing the charge parameter as $Q=q / q_{\text {ext }}$ where $Q \in[0,1]$. The analysis of the time domain profile and the quasinormal frequencies show that the Maxwellian regular black holes are stable against electromagnetic perturbations.

In Cardoso et al. (2009) it was stated that in the eikonal (high energy or large multipole number) limit quasinormal modes are characterized by the unstable circular null geodesics. In Toshmatov et al. (2018) we showed by the electromagnetic perturbations of the nonlinear electrodynamics black holes that this claim is correct in the standard linear electrodynamics. However, it does not work in nonlinear electrodynamics, since in 
the nonlinear electrodynamic field a photon does not follow the null geodesics. Instead it follows the null geodesics of an effective metric. We claim that in the eikonal regime, the quasinormal modes of nonlinear electrodynamic black holes are characterized by the unstable circular photon orbits determined by the effective geometry.

\section{Optical properties of black holes}

We have studied the shadow of a rotating black hole with nonvanishing gravitomagnetic charge Abdujabbarov et al. (2013). It was shown that in addition to the angular momentum of the black hole, the gravitomagnetic charge term deforms the shape of the black-hole shadow. From the numerical results we have obtained for a given value of the rotation parameter, the presence of a gravitomagnetic charge enlarges the shadow and reduces its deformation with respect to the spacetime without gravitomagnetic charge. We have also studied the capture cross-section for massive particles by a black hole with nonvanishing gravitomagnetic charge.

In Atamurotov et al. (2013a) we have studied the shadow of a rotating non-Kerr black hole, and it was shown that in addition to the specific angular momentum, the deformation parameter of non-Kerr spacetime essentially deforms the shape of the black hole shadow. For a given value of the black hole spin parameter $a$, the presence of a deformation parameter reduces the shadow and enlarges its deformation with respect to the one in the Kerr spacetime. Optical features of the rotating non-Kerr black hole in terms of rotation of the polarization vector along null congruences have been also studied.

The shadow of a rotating Hořava-Lifshitz black hole has been studied in our paper Atamurotov et al. (2013b) and it was shown that in addition to the specific angular momentum $a$, parameters of Hořava-Lifshitz spacetime essentially deform the shape of the black-hole shadow. We have found a dependence of radius of the shadow and distortion parameter from coupling parameters of the modified gravity.

In Atamurotov et al. (2015) we have studied photon motion around axially symmetric rotating Kerr black holes in the presence of a plasma with radial power-law density. It was shown that, in the presence of a plasma, the observed shape and size of the shadow changes depending on (i) the plasma parameters, (ii) black hole spin, and (iii) the inclination angle between the observer plane and the axis of rotation of the black hole. In order to extract the pure effect of the plasma influence on the black-hole image, the particular case of the Schwarzschild black hole has also been investigated and it has been shown that the photon sphere around the spherically symmetric black hole is left unchanged under the plasma influence; however, the Schwarzschild black-hole shadow size in the plasma is reduced, owing to the refraction of the electromagnetic radiation in the plasma environment of the black hole. The study of the energy emission from the black hole in the plasma environment shows that in the presence of the plasma, the maximal energy emission rate from the black hole decreases.

In Abdujabbarov et al. (2017a) we study the shadow of the rotating black hole with quintessential energy (i) in vacuum and (ii) in the presence of a plasma with radial powerlaw density. For the vacuum case the quintessential field parameter of the rotating black hole sufficiently changes the shape of the shadow. With increasing the quintessential field parameter, the radius of the shadow also increases. With the increase of the radius of the shadow of the rotating black hole the quintessential field parameter causes a decrease of the distortion of the shadow shape. In the presence of the quintessential field parameter, the shadow of a fast rotating black hole is starting to become more close to a circle. The shape and size of the shadow of a quintessential rotating black hole surrounded by a plasma depends on (i) plasma parameters, (ii) the black-hole spin and (iii) the quintessential field parameter. With the increase of the plasma refraction index the apparent radius of the shadow increases. However, for the big values of the 
quintessential field parameter, the change of the black-hole shadow's shape due to the presence of the plasma is not sufficient. In other words: the effect of the quintessential field parameter becomes more dominant in comparison to the effect of the plasma.

In Abdujabbarov et al. (2016a) we study the shadows cast by the different types of rotating regular black holes viz. Ayon-Beato-Garcia (ABG), Hayward and Bardeen. These black holes have in addition to the total mass $(M)$ and rotation parameter $(a)$, different parameters such as electric charge $(Q)$, deviation parameter $(g)$, and magnetic charge $\left(g^{*}\right)$. Interestingly, the size of the shadow is affected by these parameters in addition to the rotation parameter. We found that the radius of the shadow in each case decreases monotonically, and the distortion parameter increases when the values of these parameters increase. A comparison with the standard Kerr case is also investigated. We have also studied the influence of the plasma environment around regular black holes to discuss the shadow. The presence of the plasma affects the apparent size of the regular black hole's shadow to be increased due to two effects: (i) gravitational redshift of the photons and (ii) radial dependence of plasma density.

Abdujabbarov et al. (2016b) is devoted to study of the massless particle motion around a rotating wormhole in the presence of a plasma environment. It has been shown that the presence of the plasma decreases the inner radius of the circular orbits of photons around a rotating wormhole. The shadow cast by the rotating wormhole surrounded by an inhomogeneous plasma with the radial power-law density has been explored. It has been shown that the shape and size of the wormhole shadow is distorted and changed, depending on (i) plasma parameters, (ii) wormhole rotation and (iii) the inclination angle between the observer plane and the axis of rotation of the wormhole. As an example, we have considered an inverse radial distribution of the plasma density and different types of wormhole solution.

In the weak field approximation, we have studied in the paper Abdujabbarovet al. (2017b) the gravitational lensing near the regular Bardeen, Hayward and Ayon-BeatoGarcia (ABG) black holes surrounded by plasma. The exact expressions for the deflection angle of the photons due to the effect of the gravitational field and the plasma have been obtained. The analysis of the image source brightness magnification in the background spacetimes of (i) Bardeen, (ii) Hayward and (iii) ABG regular black holes have shown that the increase of the corresponding charge of a regular black hole causes the increase in the magnification of the source image. In addition to the primary ring, one may observe the secondary ring with smaller magnification. The influence of the plasma with (i) constant and (ii) radial power law electron density on the magnification of the source image has been studied.

We have aslo studied in Turimov et al. (2018) the gravitational lensing in the weak-field approximation, assuming the presence of a plasma and of a magnetic field around a compact gravitational source. The external magnetic field causes the splitting of the image, as the counterpart of the Zeeman effect. The magnetic field affects the magnification of images, creating additional components. We also study the time delay of an electromagnetic signal due to the geometry and the gravitational field around the source. We show that the time delay strongly depends on the plasma parameters. Lastly, we consider the effects of the presence of an inhomogeneous plasma on the gravitational lensing.

Much attention is paid to the graduate and post-graduate study on relativistic astrophysics in Uzbekistan. For example, in the year 2018 there were four PhD dissertations defences by the students enrolled in relativistic astrophysics study in Uzbekistan: (i) Farruh Atamurotov, Optical and energetic processes in the vicinity of rotating relativistic compact objects, March; (ii) Arman Tursunov, Astrophysical processes in the field of compact objects, June; (iii) Bobir Toshmatov, Particles and fields around compact objects, August; (iv) Sanjar Shaymatov, General relativistic astrophysical processes 
in the vicinity of compact gravitational objects in the presence of an electromagnetic field, December, which confirm the rapid growth of studies in relativistic astrophysics in Uzbekistan.

\section{Acknowledgements}

This work is supported by the Grant No. VA-FA-F-2-008 of the Ministry of Innovational Development of the Republic of Uzbekistan and by the Abdus Salam International Centre for Theoretical Physics (ICTP) through the Grant OEA-NT-01.

\section{References}

Abdujabbarov, A., Atamurotov, F., Kucukakca, Y., Ahmedov, B. and Camci, U. 2013, Astrophys. Space Sci., 344, 429

Abdujabbarov, A., Amir, M., Ahmedov, B. and Ghosh, S.G. 2016, Phys. Rev. D, 93, 104004

Abdujabbarov, A., Juraev, B., Ahmedov, B. and Stuchlík, Z. 2016, Astrophys Space Science, 361,226

Abdujabbarov, A., Toshmatov, B. Stuchlík, Z. and Ahmedov, B. 2017, International Journal of Modern Physics D, 26, 1750051-239

Abdujabbarov, A., Toshmatov, B., Schee, J., Stuchlík, Z. and Ahmedov, B. 2017, International Journal of Modern Physics D, 26, 1741011-187

Atamurotov, F., Abdujabbarov, A. and Ahmedov, B. 2013, Astrophys. Space Sci., 348, 179

Atamurotov, F., Abdujabbarov, A. and Ahmedov, B. 2013, Phys. Rev. D, 88, 064004

Atamurotov, F., Ahmedov, B. and Abdujabbarov, A. 2015, Phys. Rev. D, 92, 084005

Ayón-Beato, E. and García, A. 1998, Phys. Rev. Lett. B, 80, 5056

Bambi, C., Modesto, L. and Rachwal, L. 2017, J. Cosmol. Astropart. Phys., 05, 003

Cardoso, V., Miranda, A.S., Berti, E., Witek, H. and Zanchin, V.T. 2009, Phys. Rev. D, 79, 064016

Fan, Z.-Y. and Wang, X. 2016, Phys. Rev. D, 94, 124027

Modesto, L. and Rachwal, L. 2016, arXiv:1605.04173 [hep-th]

Toshmatov, B., Ahmedov, B., Abdujabbarov, A. and Stuchlík, Z. 2014, Phys. Rev. D, 89, 104017

Toshmatov, B., Abdujabbarov, A., Ahmedov, B. and Stuchlík, Z. 2015, Astrophysics and Space Science, 357, 41

Toshmatov, B., Abdujabbarov, A., Stuchlík, Z. and Ahmedov, B. 2015, Phys. Rev. D, 91, 083008

Toshmatov, B., Stuchlík, Z., Schee, J. and Ahmedov, B. 2016, Phys. Rev. D, 93, 124017

Toshmatov, B., Stuchlík, Z. and Ahmedov, B. 2017, European Physical Journal Plus, 132, 98

Toshmatov, B., Stuchlík, Z. and Ahmedov, B. 2017, Phys. Rev. D, 95, 084037

Toshmatov, B. and Stuchlík, Z. 2017, European Physical Journal Plus, 132, 324

Toshmatov, B., Bambi, C., Ahmedov, B., Stuchlík, Z. and Schee, J. 2017, Phys. Rev. D, 96, 064028

Toshmatov, B., Stuchlík, Z., Schee, J. and Ahmedov, B. 2018, Phys. Rev. D, 97, 084058

Turimov, B., Ahmedov, B., Abdujabbarov, A. and Bambi, C. 2018, arXiv:1802.03293 [hep-th]

Younas, A., Jamil, M., Bahamonde, S. and Hussain, S. 2015, Phys. Rev. D, 92, 084042 